\title{
Systematic Review Study of The Using of "Guided Growth" For Correction of Coronal Deformities Around The Knee in Skeletal Immature Children
}

\author{
Mohammed R. El Hosainy, Galal Kazem, Hosam Farag
}

\begin{abstract}
Department of Orthopaedic surgery, Benha faculty of medicine, Benha University, Egypt.

\section{Correspondence to: \\ Mohammed R. El Hosainy, Orthopaedic surgery department, Benha faculty of medicine, Benha University, Egypt.}

Email:

mohammed.eldebs@yahoo.com

Received: 28 August 2019

Accepted: 19 January 2020

\begin{abstract}
:
Angular deformities of the lower limbs are common during childhood. The concept of guided growth using a tension band plate is a breakthrough in the surgical correction of angular deformities. This approach has yielded satisfactory results and has low complication rates. We reviewed 210 papers with overall 433 patients and 809 deformities. Inclusion criteria included papers published from 2010 to 2017 in English literature. A Systematic review was done on 16 papers with seven outcomes: Change in mechanical axis deviation, tibiofemoral angle, rate of excellent outcomes, implant breakage, implant migration, inadequate reduction and rebound phenomenon. Thirty-two studies were included. Fifteen of them studied treatment of angular deformities by guided growth, three discussed the complications of using guided growth and five studies discussed biomechanics of using guided growth. Sixteen studies were included in our systematic review. Guided growth is effective in treating coronal deformities around the knee in skeletally immature children whether the cause was idiopathic or pathological. There is no limitation except if physeal bar is developed or the patient was near maturity. Few complications are associated with guided growth techniques including rebound phenomenon, inadequate reduction, implant breakage and implant migration.
\end{abstract}

Keywords: Guided growth; Eight plate; Staples. 


\section{Introduction:}

Guiding growth by harnessing the ability of growing bone to undergo plastic deformation is one of the oldest orthopedic principles. There has been renewed interest in surgical methods of physeal manipulation or 'guided growth'. Manipulating natural bone growth to correct a deformity is appealing, as it allows gradual correction by non-or minimally invasive methods $[1,2]$.

Heuter [1] first provided a scientific explanation for the phenomenon of mechanical manipulation of bone growth in 1862, when he reported that increased pressure parallel to the axis of the epiphysis inhibits growth, while decreased pressure promotes it.

Seven years later, Volkmann [2] noted that changes in compressive forces cause asymmetrical growth of a joint. The 'chondral modeling' theory of Frost [3] suggests that the relationship between loading and chondral growth resembles an inverted $U$ shape. Physiological loading stimulates growth, while loads outside this range, either higher or lower, will inhibit it. Thus, minor degrees of joint incongruency, where the stresses remain within physiological limits, invoke a negative feedback in order to restore the joint to normal. Increasing incongruency results in the physis being subjected to loads outside the normal physiological range, invoking a positive feedback mechanism which results in progressive deformity.

This complex, non-linear relationship has many implications for the management of deformity, including a window outside which physeal manipulation may fail. Most importantly, it suggests that any intervention should be performed at an early stage when negative feedback correction can be harnessed. Early restoration of the mechanical axis is desirable to avoid permanent abnormality of the adjacent joint surfaces, which would otherwise lead to long-term morbidity. Performing a surgical physeal arrest using permanent or potentially reversible techniques may avoid the need for osteotomies in treatment of deformities in growing children [3].

The overall problem with performing a permanent physeal arrest is the need for timing of the procedure .The technique was first introduced by Phemister [4] who described a technique to obtain permanent fusion of the growth plate by performing a rectangular resection of bone containing metaphysic, physis and epiphysis with the resected area subsequently being reinserted with the ends reversed. By using this procedure, both equalizing of leg length and correction of frontal plane deformities such as genu valgum could be achieved according to Phemister [4]. This procedure has disadvantages including 
considerable and prolonged postoperative care .One of the first attempts to perform a reversible hemiepiphysiodesis was made by Haas [5] who inserted a wire over the physis in growing dogs leading to growth arrest of the affected physis.

In 1949, Blount and Clarke reported on stapling of the epiphyseal plate as a method to correct both angular deformities and LLD [68]. Tension band plating developed to address some of the complications associated with the use of staples. It was first described by Stevens who developed the eight-plate implants (Orthofix; McKinney, TX, USA). These implants have been advocated to avoid compression of the growth plate and to reduce mechanical failures [9].

In this paper, we systematically review the literature for the using of Guided Growth techniques to correct coronal deformities around the knee in skeletally immature children, covering the technical points, the outcomes and the occurrence of complications.

\section{Methods:}

\section{Search strategy:}

The literature was reviewed through electronic databases; Egyptian Knowledge Bank (EKB), PubMed and Cochrane library about the using of guided growth for correction of coronal deformities around the knee in skeletally immature children.

We used the following keywords: Hemiepiphysiodesis, guided growth, eight plate, staples, coronal knee deformities, tension band plating, genu valgum, genu varum, growth plate and growth modulation for the search.

\section{Population, intervention, comparator, outcomes and study design}

We included studies done by randomized controlled trials (RCT) techniques, prospective studies and retrospective cohort studies with at least one data point before and after the intervention. Cross-sectional studies, expert opinion studies and case reports studies were excluded.

Skeletally immature children (at least 24 months of predicted growth were remaining) with coronal deformities around the knee (genuvalgum or genuvarum) either idiopathic or pathological physis were included. We excluded skeletally mature children, children with expected less than two years to reach maturity, physiological deformities and dynamic deformities due to ligamentous laxity as in neuromuscular disorders.

The literature search was limited to English language studies published from January 2010 till December 2017. The primary outcomes were changes in Mechanical Axis Deviation (MAD) and Tibiofemoral Angel (TFA). Secondary outcomes included 
excellent results rate, rate of implant breakage, implant migration, inadequate reduction and rebound phenomenon. This study has been reported according to the Preferred Reporting Items for Systematic Reviews (PRISMA) guide lines [9].

\section{Study selection}

A total of 210 studies were identified and imported into Mendeley Desktop V.1.17.13 Reference Manager. After removing duplicates/papers published before 2010, 63 articles underwent title screening. Studies were excluded if they were non-human $(n=2)$, nota research study $(n=14)$, flexion knee deformities $(n=4)$, Ankle/Hip deformities $(n=8)$, case reports $(n=9)$ and not in English $(n=3)$. Two independent reviewers assessed articles for eligibility; all disagreements were corrected through negotiated consensus with the third reviewer.

\section{Data Extraction}

Relevant quantitative and qualitative data necessary for adequate study comparison and analysis was abstracted.

Studies should contain these items: objective, design, type of intervention, changes in Mechanical Axis Deviation (MAD) and Tibiofemoral Angel (TFA), excellent results rate, rate of implant breakage, implant migration, inadequate reduction and rebound phenomenon.

\section{Limitations}

Only two RCT were published, Most of the papers were single arm (not comparing guided growth with corrective osteotomy or staples with eight plate), Only one paper used Mechanical Axis Ratio for evaluation of outcomes, presence of different modalities of evaluation of the primary outcomes (MAD, TFA, mLDFA, mMPTA, etc), Sub-grouping differes from paper to another (pathological /idiopathic, genuvarum/ valgum, Age, BMI).

\section{Results}

Twenty-three studies were included in our systematic review. Fifteen studied treatment of angular deformities by guided growth, three discussed the complications of using guided growth and five discussed Biomechanics of using guided growth around the knee.

\section{Discussion}

In our Systematic review 433 patients with 809 deformities undergone different procedures for treatment of coronal angular deformities around the knee using the concept of guided growth by staples or titanium eight plates or stainless steel eight plates or stainless steel one third tubular plates. Guided growth techniques could be used in skeletally immature children provided that there is at least one-year growth remaining and there is no physeal bar developed in the physis. 
Eight plate was studied by Vaishya et al [1],Baghel [12], Heflin [13], Kulkarni [14], Ashby [15], Das [16], Stevens [9], Boero [17] and Ballal [18]. They found that it is effective in treating coronal angular deformities around the knee.

Pathological physis was studied by Boero [17], Ashby [15] and Heflin [13]. They agreed that Idiopathic deformities get more benefit of guided growth than pathological one. Our systematic review showed that TFA corrected by 4.39 SMDin idiopathic groups while in pathological groups was corrected by 2.38SMD. It's better to start earlier intervention by guided growth in pathological physis as the speed of correction is slow in comparison with the idiopathic group and also to prevent or even reverse secondary issues such as ligamentous laxity, lateral sublaxation and torsion or recurrence of deformity.

On the opposite side Colmenares-bonilla [19] recommends corrective osteotomy in treating patients with Morquio-A (MPS) because they usually had insufficient growth power especially when been diagnosed in adolescent age and this may help reduce arthritis progression in adjacent joints.

As we have different methods for the application of guided growth concept, Hosseinzadeh [20] and Kumar [21] studied the difference in the different constructs. Stainless steel constructs have higher rate of correction that explain its increased strength for angular correction. The stainless steel may increase the relative shear strength of that implants they have not seen any hardware failures with the stainless

Steel Eight plates. Eight plates have higher rate of correction when compared to staples.

On the opposite side, the study of Gottliebsen [22] reported that there is no difference in treating angular deformities with eight plate or staples as regards the results of correction of the deformities but, this study was under powered because of small number of population .Lin [23] proposed a different way for guided growth concept using two holes one third tubular plate and it showed excellent results in treating angular deformities around the knee. It could be used in developing countries where there are no eight plates available.

Post operatively Fillingham recommends immediate postoperative physiotherapy as it prevents significantly the delay in return of function.

Our systematic review showed that Rebound Phenomenon rate was $4 \%$ in eight plate group and $6 \%$ in staples group.

Leveille [25], Kang [26] and Burghardt [27] modulation at a young age $(<10 \& 12$ years in girls and boys respectively) and those with large initial deformities (>20 degrees 
mechanical axis deviation from neutral).They also found that when we have a rate of correction $\geq 8.5^{\circ} /$ year and a Body mass index $<21 \mathrm{~kg} / \mathrm{m} 2$, rebound phenomenon is highly recommend. Over correction especially when we have growth anticipated. Mechanical failure also occurs most probably in the metaphyseal screw not where the head meets the shank but where the shank enters the lateral cortex. Our systematic review showed that the rate of implant breakage was $4 \%$ in eight plate group while in staples group was $3 \%$.

Leveille [25], Kang [26] and Burghardt [27] bending of the plate and alternately tightening the 2 screws. It is advised that screws should not be placed widely divergent because it may impinge on the plate prematurely. They also recommend the using of solid screws rather than cannulated screws. Screw breakage was reported in patients with an average BMI of $37.8 \mathrm{~kg} / \mathrm{m} 2$ range (19.2 to $70.2 \mathrm{~kg} / \mathrm{m} 2)$, that's why, it is recommended to use two parallel eight-plates in either a parallel or oblique fashion. Staples have higher risk of migration/extrusion than the other constructs because of the fulcrum being extraperiosteal. Our systematic review showed that the rate of implant migration was $2 \%$ in eight plate group and $8 \%$ in staples group.

In distal medial femoral hemiepiphysiodesis, Bachmann [28] reported that there is a risk in injuring MPFL but not the MCL which explain the excessive postoperative pain that sometimes occur together with reduced ROM after temporary hemiepiphysiodesis at distal medial femur with a plate and two screws.

Gyr[29] studied treating angular deformities in pediatric amputees using the means of guided growth which was successful and avoided the complications of using external or internal fixation devices.

Marangoz [30] documented that there is correlation, between the delta joint orientation angle and the delta inter screw angle. This correlation is not related to the growth rate of the physis. It is a purely mathematical correlation, independent of the age and sex of the patient. It can be used to measure the anatomical changes of the joint line without the need of full length X-rays.

Ceroni [31] studied the modification of the alignment between the tibial tubercle (TT) and trochlear groove (TG) that occur during guided growth. They stated that for every degree of angular correction recommend that the plate should be coopted to the bone by pre during femoral distal hemiepiphysiodesis, there is a $1 \mathrm{~mm}$ simultaneous lateral or medial transfer of the TT. And during proximal tibial hemiepiphysiodesis, $8^{\circ}$ of angular correction will roughly translatetheTTby $1 \mathrm{~mm}$. 
Masquijo [32] studied the modified Paley technique. It seems that this technique reduces operative time, radiation exposure, and incision size for guided growth around the knee.

\section{Conclusion}

Guided growth is effective in treating coronal deformities around the knee in skeletally immature children wither the cause was idiopathic or pathological. There is no limitation except if physeal bar is developed or the patient near maturity. Few complications are associated with guided growth techniques including rebound phenomenon, inadequate reduction, implant breakage and implant migration.

\section{References:}

1. Hueter C (1863) Anatomical studies one xtremity joints newborn and adult.Arch Pathol Anatom Physiol ClinMed26:484-519.

2. Volkmann $\mathrm{R}$ (1869) The diseases of the locomotor organs.Billroth Pitha,edMan Gen Spec Surgery Stuttgart Ferdinand Enke 2:694.

3. FrostHM(1979)Achondralmodelingtheory.CalcifiTis suIntern28:181-200.

4. Phemister DB (1933) Operative arrestment of longitudinal growth of bones in the treatment of Deformities.JBoneJtSurg 15:1-15.

5. Haas SL(1945)Retardation of bone growth by a wire loop.JBoneJtSurgAm27:25-36.

6. Moher D, Liberati A, TetzlaffJ, Altman DG, PRISMA Group (2009) Preferred reporting items for systematic reviews and meta- analyses:The
PRISMA statement. BMJ 339:2535.

7. Stevens PM, Novais EN(2012)Multilevel guided growth for hip and knee varus secondary to chondrodysplasia.JPediatrOrthop32:626-630.

8. Blount WP, Clarke GR (1949) Control of bone growth by epiphyseal stapling:A preliminary report.JBoneJtSurgAm31: 464478.

9. Stevens $P M(2007)$ Guided growth for angular correction:A preliminary series using a tension band plate.JPediatrOrthop 27:253-259.

10. Duval S,Tweedie R(2000)Trim and fill:A simple funnel-plot- based method of testing and adjusting for publication bias in metaanalysis.Biometrics56:455-463.

11. Vaishya R, Shah M, Agarwal AK, Vijay V (2017) Growth modulation by hemiepiphysiodes is using eight-plate in Genu valgum in paediatric population. JClinOrthop Trauma(A

12. BaghelA,AgrawalA,SinhaS,SinghRP,Shar maV, et al. (2016) Evaluation of rate of correction of angular deformity of knee using tension band plate. Intern J Contemp Med Res 3: 2454-7379.

13. Heflin JA,Ford S,Stevens P(2016)Guided growth for tibia vara (Blount's disease).Medicine (Baltimore)95:e4951.

14. Kulkarni RM, Rushnaiwala FMI, Kulkarni GS, Negandhi R,Kulkarni MG, (2015) Correction of coronal plane deformities around the knee using a tension band plate in children younger than 10 years Indian $\mathrm{J}$ Orthop 49:208-218.

15. Ashby E,Eastwood D(2015)Characterization of knee alignment in children with mucopolysaccharidosis types I and II and outcome of treatment with guided growth.JChildOrthop9: 227-233. 
16. Das S, Ganesh GS, Pradhan S, Mohanty RN (2014) Outcome of eight-plate hemiepiphysiodesis on genu valgum and height correction in bilateral fibularhemimelia. $\mathrm{J}$ Pediatr Orthop 23: 67-72.

17. Boero S,Michelis MB,Riganti S(2011)Use of the eight-plate for angular correction of knee deformities due to idiopathic and pathologic physis: Initiating treatment according to etiology J Child Orthop5:209-216.

18. Ballal MS, Bruce CE, Nayagam S (2010) Correcting genu varum and genu valgum in children by guided growth :Temporary Hemiepiphysiodesis using Tension Band Plates. J Bone JtSurg92:273-276.

19. Colmenares-Bonilla D,Vasconcelos-Martinez M,Guerra-Jasso J (2017) Be the best option for knee valgus deformity in adolescent patients with Morquio-A. Musculoskelet Surg101:113-118.

20. Hosseinzadeh P,D. R. Ross DR,Walker J. L.Walker,Talwalkar VR,Iwinski HJ,T.A Milbrandt et al.(2016)Three methods of guided growth for pediatric lower extremity angular deformity correction. Iowa Orthop J 36: 123 127.

21. Kumar A, Gaba S, Sud A, Mandlecha P, Goel L.(2016) Comparative study between staples and eight plate in the management of coronal plane deformities of the knee in skeletally immature children.J ChildOrthop10:429-437.

22. Gottliebsen M, Rahbek O, Hvid I, Davidsen M, Hellfritzsch MB. (2013) Hemiepiphysiodesis: Similar treatment time for tension-band plating and for stapling:A randomized clinical trial on guided growth for idiopathic genu valgum.ActaOrthop84: 202-206.

23. Lin TY, Kao HK, Li WC, Yang WE, Chang CH (2013)Guided growth by a stainless-steel tubular plate.JPediatrOrthop22:306-310.
24. Fillingham YA, Luthringer T, Erickson BJ, Kogan M (2015) Doesphysical therapy prevent postoperative delay in return of function following tension-band plating? J Child Orthop 9: 483487.

25. Leveille LA, Razi O, Johnston CE (2017) Rebound deformity after growth modulation in patients with coronal plane angular deformities about the knee: Who gets it and how much? J PediatrOrthop 98:37.

26. Kang S, Kim JY, Park SS (2016) Prediction of reboundphenomenon after removal of hemiepiphyseal staples in patients with idiopathic genu valgum deformity. Bone Jt J 98:1270-1275.

27. Burghardt RD, Specht SC, Herzenberg JE (2010) Mechanical failures of eight-plate guided growth system for temporary hemiepiphysiodesis. J Pediatr Orthop30:594597.

28. Bachmann M, Rutz E, Brunner R, Gaston MS, Hirschmann MT, et al. (2014) Temporary hemiepiphysiodesis of the distal medial femur: MPFL in danger. Arch Orthop Trauma Surg 134:1059-1064.

29. Gyr BM, Colmer HG, Morel MM, Ferski GJ (2013) Hemiepiphysiodesis for correction of angular deformity in pediatricamputees. J PediatrOrthop33:737-742.

30. Marangoz S, Buyukdogan K, Karahan S (2017) Is there a correlation between the change in the interscrew angle of the eight-plate and the delta joint orientation angles? Acta Orthop Traum atolTurc51:39-43.

31. Ceroni D, Dhouib A, Merlini L, Kampouroglou G (2017)Modification of the alignment between the tibial tubercle and the trochlear groove induced by temporary hemiepiphysiodesis for lower extremity angular deformities: A trigonometric analysis. J Pediatr Orthop 26:204-210. 
32. Masquijo JJ, Lanfranchi L, Torres-Gomez A ,Allende $\mathrm{V}(2015)$ Guided growth with the tension band plate construct :A prospective comparison of 2 methods of implant placement. J PediatrOrthop35:20-25.

To cite this article: Mohammed R. El Hosainy, Galal Kazem, Hosam Farag Systematic review study of the using of Guided Growth for correction of coronal deformities around the knee in skeletally immature children, BMFJ, 2020; 37(2), 382390. DOI:10.21608/bmfj.2020.16358.1043 